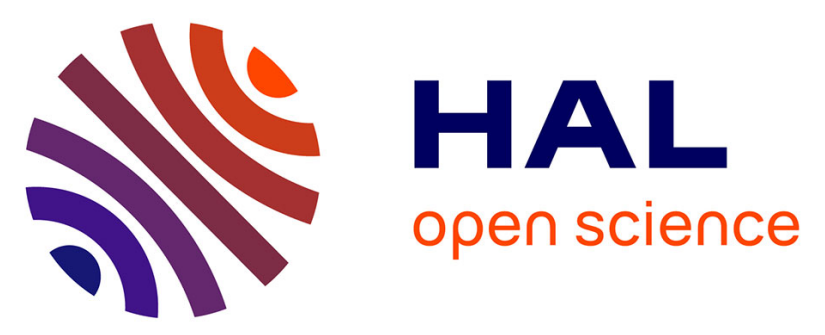

\title{
Essai de cartographie intégrée d'un espace forestier : la forêt de Bouconne
}

Gerard Herail, Jacques Hubschman

\section{To cite this version:}

Gerard Herail, Jacques Hubschman. Essai de cartographie intégrée d'un espace forestier: la forêt de Bouconne. Revue Géographique des Pyrénées et du Sud-Ouest, 1978, 49, 2 (221-238), 10.3406/rgpso.1978.3551 . hal-02862128

\section{HAL Id: hal-02862128 \\ https://hal-univ-tlse2.archives-ouvertes.fr/hal-02862128}

Submitted on 9 Jun 2020

HAL is a multi-disciplinary open access archive for the deposit and dissemination of scientific research documents, whether they are published or not. The documents may come from teaching and research institutions in France or abroad, or from public or private research centers.
L'archive ouverte pluridisciplinaire HAL, est destinée au dépôt et à la diffusion de documents scientifiques de niveau recherche, publiés ou non, émanant des établissements d'enseignement et de recherche français ou étrangers, des laboratoires publics ou privés. 

Bouconne

Gérard Hérail, Jacques Hubschman

\section{Citer ce document / Cite this document :}

Hérail Gérard, Hubschman Jacques. Essai de cartographie intégrée d'un espace forestier : la forêt de Bouconne. In: Revue géographique des Pyrénées et du Sud-Ouest, tome 49, fascicule 2, 1978. Géosystème et aménagement. pp. 221238;

doi : https://doi.org/10.3406/rgpso.1978.3551

https://www.persee.fr/doc/rgpso_0035-3221_1978_num_49_2_3551

Fichier pdf généré le 13/09/2018 


\begin{abstract}
The Bouconne forest near Toulouse. An essay of an integrated mapping of a woodland area. Grown west of Toulouse on the highest alluvial terraces built up by the Garonne river, the Bouconne woodland area is commonly known as a forest when it is an oak taillis dottered with pine trees. The spatial distribution of the various woodland geofacies and the growth of the aggregate vegetal biomass as well depend on one hand on the soils' nature (and mainly on their drainage) and on the other hand on the economic management of the woodland and the various crisis that it met (wood fires, invasions of parasites or too frequent cutting down of timber on the same plot). Drainage and timber growth quickness are the two major keywords for the map.
\end{abstract}

\title{
Résumé
}

La forêt de Bouconne, située sur les hautes terrasses de la Garonne à l'ouest de Toulouse, est un taillis de Chênes piqueté de Pins. La distribution des divers géofaciès forestiers et la croissance de la biomasse forestière sont fonction de la qualité des sols (surtout de leur drainage) d'une part, de la gestion et des crises (incendies, parasites, coupes parfois très rapprochées) d'autre part. Mais en dépit des aménagements l'aspect et la dynamique des peuplements forestiers restent étroitement tributaires des grandes variables naturelles et surtout du régime hydrique des sols. Ces contrastes ont été renforcés par la succession des opérations d'aménagement et c'est le parallélisme entre le bon drainage du sol et la rapidité de croissance des peuplements qui est la clef de la légende de la carte.

\section{Resumen}

La "forêt de Bouconne » : un mapa forestal. La " Forêt de Bouconne " situada sobre las altas terrazas del río Garona, al oeste de Toulouse, es un monte alto de robles salpicado de pinos. La distribución de los diversos geofacies forestales y el crecimiento de la biomasa forestal están relacionados por una parte con la calidad de los suelos (esencialmente el drenaje) y por otra parte con la gestion y las crisis (incendios, parásitos, cortes a veces muy frecuentes). Pero a pesar de las ordenaciones, el aspecto y la dinámica de las poblaciones forestales quedan estrechamente ligadas a las grandes variables naturales y sobre todo el regimen hídrico de los suelos. Estos contrastes han sido acentuados por una serie de operaciones de ordenación y el paralelo que existe entre un buen drenaje del suelo y la rapidez del crecimiento de los árboles ha servido de clave para la legenda del mapa. 


\title{
Essai de cartographie intégrée d'un espace forestier : la forêt de Bouconne
}

\author{
par Gérard Hérail * et Jacques Hubschman * *
}

La cartographie forestière s'est longtemps limitée à la représentation de phénomènes isolés ou de combinaisons élémentaires (c'est le cas, en particulier, des travaux botaniques, phytosociologiques, pédologiques, etc.), quand elle n'était pas, plus simplement encore, exclusivement destinée à situer dans l'espace les unités d'exploitation et les secteurs d'intervention (parcelles de reboisement, aires d'éclaircissage, etc.). Cartes plutôt analytiques, donc presque toujours strictement accordées à un objcctif déterminé de l'inventaire scientifique ou de la gestion forestière, elles ne donnent de la forêt qu'une image fragmentaire : collections précises d'individus, d'espèces ou de parcelles, plus que transcriptions, même imparfaites, de l'ensemble du milieu forestier. D'où l'idée, encore relativement peu répandue à l'époque (1), de tenter une cartographie forestière intégrée, représentation dans laquelle les faits de structure apparaîtraient clairement, sans occulter pour autant les données constitutives majeures.

Pour ce premier essai, le choix s'est porté sur la forêt de Bouconne, proche de Toulouse. Hormis la proximité du terrain, un tel choix n'est pas celui de la facilité : exception faite de quelques net-

* Assistant de géographie à l'Université de Toulouse-Le Mirail.

** Maître-assistant de géographie à l'Université de Toulouse-Le Mirail.

1. L'étude date de 1970 et avait donné lieu à un mémoire de Maîtrise de Géographie (G. Hérail, M. Mabru, A. Machat, D. Marchio, Ecologie et aménagement de la forêt de Bouconne, Institut de Géographie, Université de Toulouse-Le Mirail). La carte présentée ici en est directement tirée; elle a cependant été légèrement remaniée depuis en vue de l'impression. Nous adressons nos plus vifs remerciements à M. Mallet, Directeur adjoint de l'ONF à Toulouse et à M. Nesson, chef de district de la forêt de Bouconne. 
tes et évidentes ruptures majeures, le tissu forestier de Bouconne ne se montre guère différencié. Dans ce massif de plaine établi sur un substrat globalement peu diversifié, les structures de l'espace forestier ne s'imposent pas clairement à l'observateur, quand bien même il en pressent l'existence. De plus, il s'agit essentiellement d'une forêt domaniale dont le dense et rigoureux parcellaire interfère fréquemment avec le zonage recherché. Tout le problème fut, précisément, d'objectiver d'abord, de classer et combiner ensuite les variables naturelles ou non, qui discriminent les unités structurales, relativement à l'échelle choisie.

Sans doute la carte ainsi conçue n'apporte-t-elle guère d'informations véritablement nouvelles sur la forêt. Son intérêt réside plutôt dans la méthode utilisée, qui vise d'abord à révéler et à classer les discontinuités de l'espace forestier, et dans le mode de représentation graphique qui cherche à transcrire au mieux les discontinuités et la logique de leur distribution. A cet égard, c'est le dynamisme des milieux qui semble le plus propre à traduire simultanément la physionomie et le déterminisme des unités forestières, comme l'indique le titre même du document.

Toute carte de ce genre constitue un système normatif, à la fois descriptif et explicatif, qui appelle le commentaire. Après une présentation des grands traits de l'espace forestier et l'analyse des variables qui déterminent la structure actuelle, on justifiera le principe du zonage et le choix des paramètres cartographiques; on examinera ensuite rapidement les unités forestières majeures mises en évidence.

\section{Une chênaie périurbaine dégradée}

A une vingtaine de kilomètres à l'ouest de Toulouse, la forêt de Bouconne s'étend sur environ 2300 ha des hautes terrasses de la Garonne. C'est, pour l'essentiel, un taillis dans lequel le Chêne pédonculé, le Chêne pubescent et le Chêne sessile composent le fond de la végétation. Quelques rares pieds de Chêne liège, dont H. Gaussen a d'ailleurs contesté le caractère spontané, poussent çà et là. Localement le Charme, qui n'est pas loin ici de sa limite méridionale, se mêle au Chêne de même qu'un certain nombre d'espèces ubiquistes : Frène, Aulne glutineux, Alisier torminal... Des essences subspontanées ou introduites, Châtaigner, Robinier faux acacia, Chêne rouge d'Amérique et divers résineux (Pin maritime et Pin sylvestre surtout) poussent en mélange avec la chênaie ou forment des îlots monospécifiques peu étendus. Dans le sous-bois, la strate herbacée est assez diversifiée et les buissons et arbrisseaux sont nombreux : Petit-houx, Ronce, Prunier, Genévrier commun, d'une part; Callune, Genêt et Bruyère à balai, d'autre part, 
Mais cette diversité floristique, d'ailleurs toute relative, n'efface pas une grande monotonie d'ensemble (2).

Dans le secteur nord-est, un taillis bas, sur une surface plane coupée de petits talus, constitue une formation où les arbres dépassent rarement 6 mètres de haut; par contre, le sous bois est toujours dense, recouvrant presque entièrement le sol. Il s'agit le plus souvent d'une chênaie dégradée claire de Chênes pédonculés associés à des Chênes pubescents. Au nord et surtout au SW, sur le palier supérieur de la terrasse, on observe la présence de Charmes plus hauts et plus vigoureux. La strate arbustive est presque inexistante, l'Alisier torminal en est l'unique représentant et quelques Sorbiers domestiques le remplacent sur les paliers les plus élevés. Par contre, la strate frutescente et herbacée, toujours fermée, est représentée par des Ronces mêlées à la Callune, à la Bruyère à balai et à un petit nombre de Graminées. L'Ajonc d'Europe et le Genêt à balai s'associent aux plantes du sous-bois quand n'apparaissent pas sur les surfaces les plus planes, les longues tiges de la Molinie bleue. Sous les Charmes, la strate frutescente et herbacée (Fragon, Lierre, petite Pervenche, Pulmonaire, Graminées, Mousses) est dominée directement par la strate arborescente, plus haute que celle de la chênaie $(10-12 \mathrm{~m})$.

Sur cette basse terrasse, le taillis médiocre et bas cède la place, çà et là, à une formation plus basse encore, la lande acide héliophile: formation temporaire, due à une ouverture récente du tapis végétal par des plantations, des incendies, des coupes qui ont détruit la chênaie claire. Cette lande impénétrable, de 2 à $3 \mathrm{~m}$ de haut, est surtout composée de plantes colonisatrices telles que la Callune, la Bruyère à balai, les Ronces, la Bourdaine ainsi que le Genêt à balai.

Au sud-ouest de la route Toulouse-Lévignac (CD 24), un second niveau $(230-235 \mathrm{~m})$, légèrement disséqué par de petits ruisseaux intermittents, présente des paysages plus contrastés.

La hauteur générale de la formation arborescente y est supérieure à celle de la terrasse du NE et les arbres y atteignent souvent 10 et $12 \mathrm{~m}$ de haut. L'uniformité de la basse terrasse, tant dans le peuplement que dans la physionomie, cède ici la place à une certaine variété liée en grande partie à la topographie. La chênaie à Chênes pédonculés est piquetée de Pins maritimes (essence introduite). Sur les plateaux, le Pin maritime domine le Chêne pédonculé, mais ce résineux n'est jamais en formation homogène et n'a guère influencé la nature du sous-bois : Ronce, Troëne, Aubépine, Sorbier domestique, Bourdaine, Chèvrefeuille, Germandrée, Lierre, Bruyère à balai et même Callune en bordure des layons. Les vallons du ruisseau de

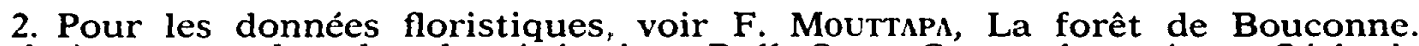
Relations entre le sol et la végétation, Buil. Serv. Carte phytogéogr., Série A, t. VI, fasc. 1, 1962. 
Fayolle et surtout le talus séparant les deux terrasses présentent un peuplement beaucoup plus vigoureux de Chêne pédonculé. Des arbres de plus de $12 \mathrm{~m}$ de haut dominent un sous-bois clair à Fougère-aigle, Lierre et Muscinées.

Au sud de la route de Léguevin à Lasserre (CD42), les plateaux sont plus élevés $(275 \mathrm{~m})$ et plus disséqués, les milieux sont plus variés.

Dans cette partie de la forêt, résineux et feuillus tendent à s'équilibrer : la chênaie à Chêne pédonculé et Chêne sessile est associée au Pin sylvestre et au Pin maritime. Quel que soit le site morphologique, palier de terrasse, talus de raccordement, vallons des ruisseaux, la strate arborée a une hauteur toujours égale ou supérieure à 12 mètres. Dans cette zone, l'impression de taillis bas et mal venu s'estompe. A l'exception de la plus basse des terrasses, où n'existe aucune formation pure de résineux, le Pin sylvestre et surtout le Pin maritime forment ici des pinèdes pures qui ont éliminé le sous-bois dense et relativement riche de la chênaie primitive (Fougère aigle, Lierre, Germandrée, Chèvrefeuille, Polygonatum vulgaire) pour le réduire à quelques Fougères aigle et à des Mousses.

\section{Une forêt sur de vieilles terrasses aux sols mal drainés}

Pour une forêt de plaine aussi peu étendue que celle de Bouconne, il est clair que la différenciation écologique exprime d'abord les modalités du substrat et de l'aménagement. Toutefois, sans être particulièrement hostiles à l'arbre, les paramètres climatiques ne restent pas sans effet, en raison notamment de la nature du sol.

\section{Un climat peu contraignant.}

Le massif reçoit en moyenne $680 \mathrm{~mm}$ d'eau pour une température moyenne annuelle de $12,7^{\circ} \mathrm{C}$. L'hiver est humide, mais, après un léger creux en février-mars, c'est au printemps que les précipitations sont les plus abondantes. Il n'existe pas à proprement parler de saison vraiment sèche (au sens de $\mathrm{H}$. Gaussen) : en juillet, mois le plus chaud avec $21^{\circ} \mathrm{C}$ de température moyenne, il tombe encore près de $40 \mathrm{~mm}$ de pluie. Toutefois, cette subsècheresse peut se faire sentir dès la fin juin et se prolonger parfois assez tard en automne. Au total, on a donc affaire à un climat plutôt modéré qui ne peut être considéré comme particulièrement défavorable à la forêt (3). Beaucoup plus contraste, en revanche, est le pédoclimat

3. Sur les problèmes climatiques, voir R.BRUNET, Les campagnes toulousaines. Toulouse, 1965, Public. Fac. Lettres de Toulouse (Série B, 1). 
qui tient à la nature du sol et, en premier lieu, aux caractères du substrat alluvial et dont le rythme amplifie considérablement les fluctuations saisonnières de l'humidité.

\section{Trois nappes alluviales étagées.}

a. Des alluvions anciennes fortement altérées. La forêt s'étend sur les trois niveaux supérieurs de l'édifice alluvial garonnais, datés respectivement du Donau (T1), Günz (T2) et Mindel (T3). Ces terrasses sont modelées en plusieurs paliers, notamment $\mathrm{T} 2$, avec deux niveaux, et surtout $\mathrm{T} 3$, la plus basse, formée de trois vastes replats. La majeure partie du substrat est constituée par d'épais cailloutis alluviaux (surtout quartz, granites et gneiss, quartzites) qui recouvrent la molasse. Mais leur caractère dominant, qui détermine pour une large part les paramètres pédologiques et hydrodynamiques de la forêt, réside dans l'altération qui affecte ces dépôts, d'autant plus poussée que les terrasses sont anciennes. Grossière et perméable à l'origine, la matrice de ces alluvions s'est transformée en un matériau argileux et colmaté qui ralentit le drainage vertical du sol. La présence régulière d'une couverture limoneuse amplifie encore ces phénomènes.

b. Des terrasses couvertes de limons. L'épaisseur de ces limons varie considérablement d'un point à l'autre : ils font parfois défaut, très localement, mais peuvent atteindre ailleurs 1 ou $2 \mathrm{~m}$, quelquefois plus. Le plus souvent, l'épaisseur de la couche se tient entre $50 \mathrm{~cm}$ et $120 \mathrm{~cm}$. Il s'agit d'un matériel fin où argiles, limons et sables très fins composent généralement 70 à $80 \%$ de la masse. Les limons colluvionnés sur les pentes sont toutefois moins homogènes, plus fréquemment mêlés de cailloutis remaniés des graves, que les dépôts recouvrant des surfaces planes. L'essentiel de ce matériel est d'origine molassique et correspond à la phase d'achèvement de chaque édifice alluvial, la mise en place étant complétée par des remaniements par ruissellement. Les limons de ces trois terrasses sont donc des formations déjà fort anciennes et les sols qui en dérivent portent la marque des longues évolutions subies par les dépôts limoneux de surface (4).

\section{Une mosaïque de sols marqués par I'hydromorphie.}

a. Trois facteurs se combinent pour imposer aux sols de la forêt un faciès très général d'hydromorphie. La grande extension des surfaces planes explique la faiblesse du drainage externe. La nature de la couverture limoneuse, fine, acide et à forte instabilité

4. J. Hubschmas, Morphogénèse et pédogénèse quaternaires dans le piémont des Pyrénées garonnaises et ariégeoises. Paris, Honoré Champion, 1975 (Thèse, Gćographie, Toulouse-Le Mirail). 746 p. 
TABLEAU

ORGANISATION DES SOLS

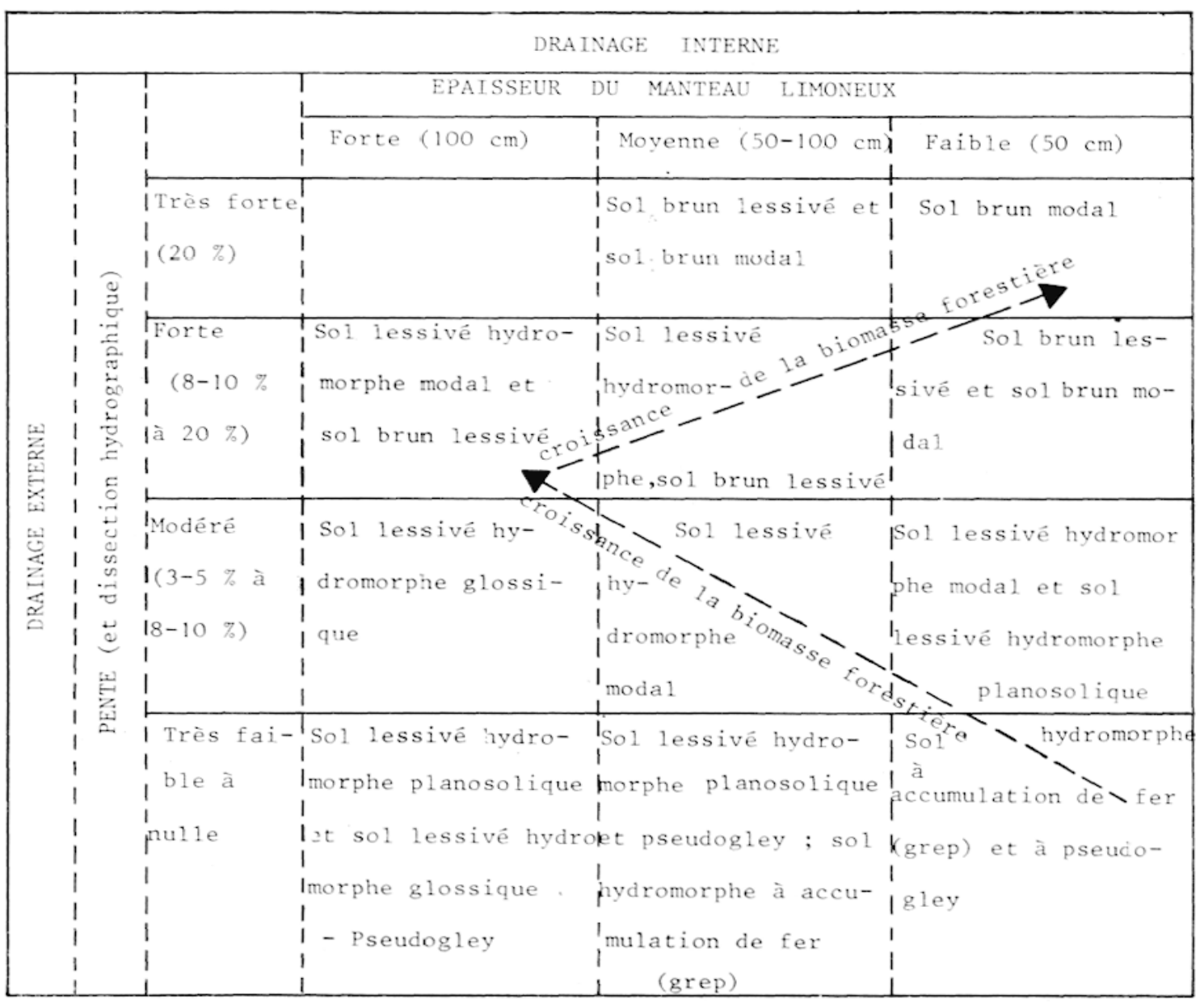

Les appellations sont ici, à quelques nuances près, celles de la Commission de pédologie et cartographie des sols (Classification des sols, document du Laboratoire de géologie-pédologie, Grignon); le pseudogley désigne les sols hydromorphes à pseudogley, dans lesquels une longue saturation par l'eau affecte très nettement lensemble du profil depuis la surface (A1 et A2 à forte redistribution du fer). Le terme modal a été introduit pour distinguer les sols lessivés hydromorphes "classiques" des variantes glossiques (horizon de " dégradation " A2g/Btg et organisation subverticale en langues profondes de redistribution du fer) et des variantes planosoliques (contact abrupt des A2g sur les Btg). (Erratum : lire pente modérée.)

structurale est peu propice à une percolation rapide. L'argilisation et le colmatage, dus à l'altération des cailloutis alluviaux sousjacents, forment un plancher peu perméable et ralentissent le drainage interne.

Les sols développés dans ce substrat ont hérité de ce dernier leurs principaux caractères. Ils restent nettement acides ( $\mathrm{pH} 4$ à 5 dans les horizons A1) et fortement désaturés (taux de saturation 
de 10 à $60 \%$ dans des horizons A2). La matière organique est relativement peu abondante pour des sols forestiers ( 3 à $4 \%$ en moyenne dans les horizons A1 par ailleurs peu épais) : la structure des horizons organiques, comme leurs propriétés chimiques, sont le plus souvent celles d'un humus de type mull-moder. Les profils montrent généralement la forte différenciation texturale propre aux sols lessivés, avec des Bt argiliques d'autant plus compacts qu'ils se développent souvent, en profondeur, dans le cailloutis argilisé. Cette différenciation marquée ajoute encore aux difficultés du drainage interne. Enfin, dans ces milieux acides et temporairement engorgés, le fer se redistribue facilement, visualisant en quelque sorte les types de sols et provoquant, par endroit, la formation d'horizons concrétionnés (grep) qui renforcent d'autant l'imperméabilité ambiante. Cependant, le modelé et la structure du substrat ont déterminé un certain nombre de variations dans les caractères du manteau de sols.

\section{L'organisation complexe de la couverture pédologique.}

Seule une cartographie à grande échelle (1/5 000 ou $1 / 10000)$ permettrait la connaissance précise de cette organisation. On se borne donc ici à une typologie grossière, fondée surtout sur la morphologie des profils, laquelle reflète le pédoclimat et notamment le degré d'hydromorphie (5).

Deux variables majeures commandent l'organisation et la distribution du manteau pédologique : la pente, qui règle le drainage externe (évacuation de l'eau hors du pédon), et l'épaisseur de la couverture limoneuse, qui détermine pour une bonne part le drainage interne. A ces deux variables s'ajoute le degré de dissection hydrographique qui intervient notablement dans le drainage interne (évacuation latérale), mais ce facteur offre une forte corrélation avec la variable pente, à laquelle il peut s'intégrer. De la combinaison de ces variables résulte un modèle simplifié d'organisation (tableau 1).

D'une façon générale, l'hydromorphie croît selon la séquence : Sol brun modal - sol brun lessivé - sol lessivé hydromorphe modal ou glossique - sol lessivé hydromorphe planosolique pseudogley - sol hydromorphe à grep. Sur les surfaces planes et les pentes médiocres, l'hydromorphie s'exprime d'autant moins que l'épaisseur du manteau limoneux est forte. Pour les pentes fortes en revanche, on observe un effet contraire : les sols sont plutôt mieux drainés lorsque les dépôts limoneux sont minces.

5. On laisse également de côté l'aspect purement génétique de la pédogénc̀sc qui peut conduire à d'autres interprétations ou désignations : ainsi les horizons développés dans les cailloutis peuvent apparenter fréquemment les profils à des sols fersiallitiques lessivés (cf. J. HuBschman : Morphogénèse et pédogénèse...). 


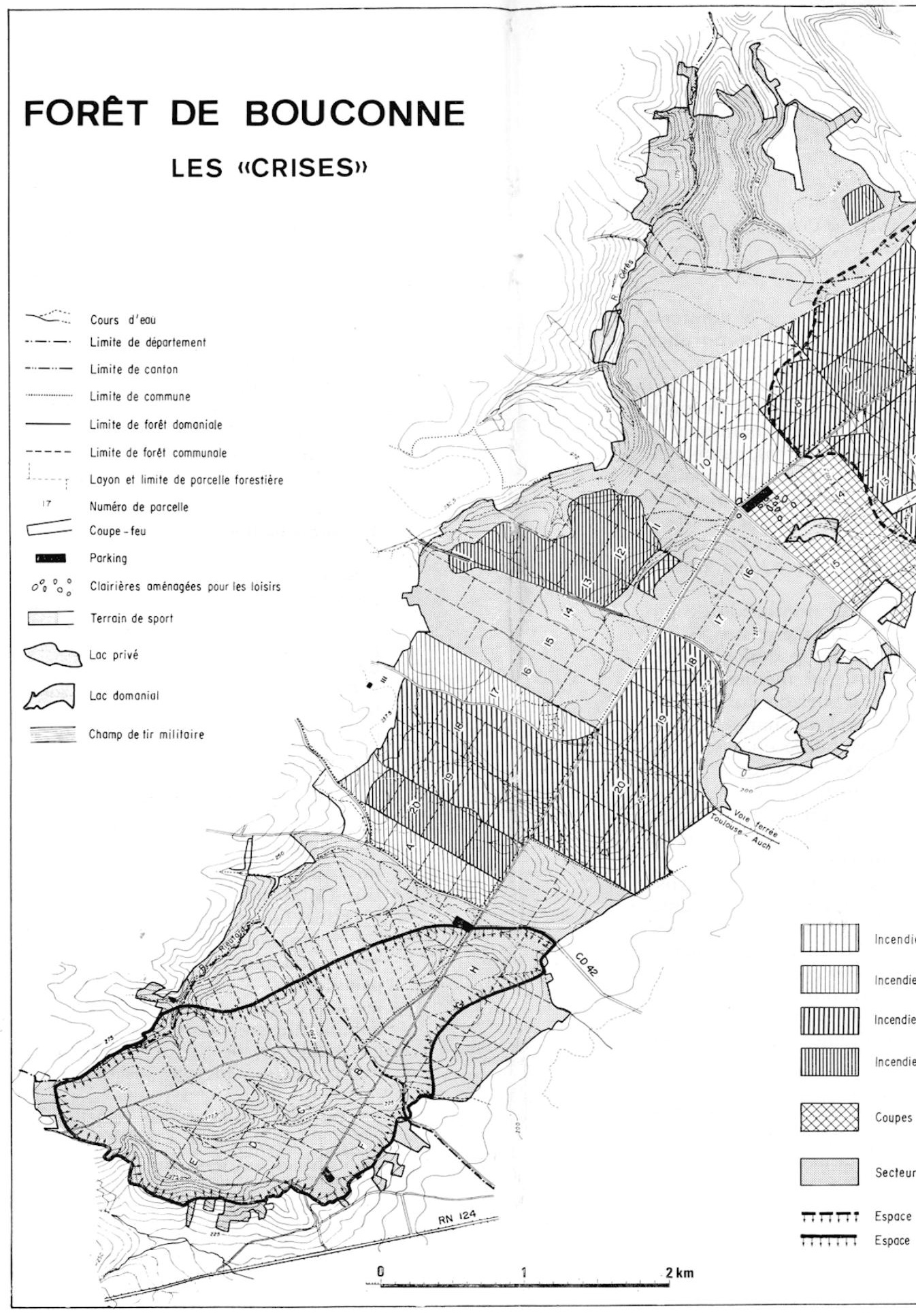




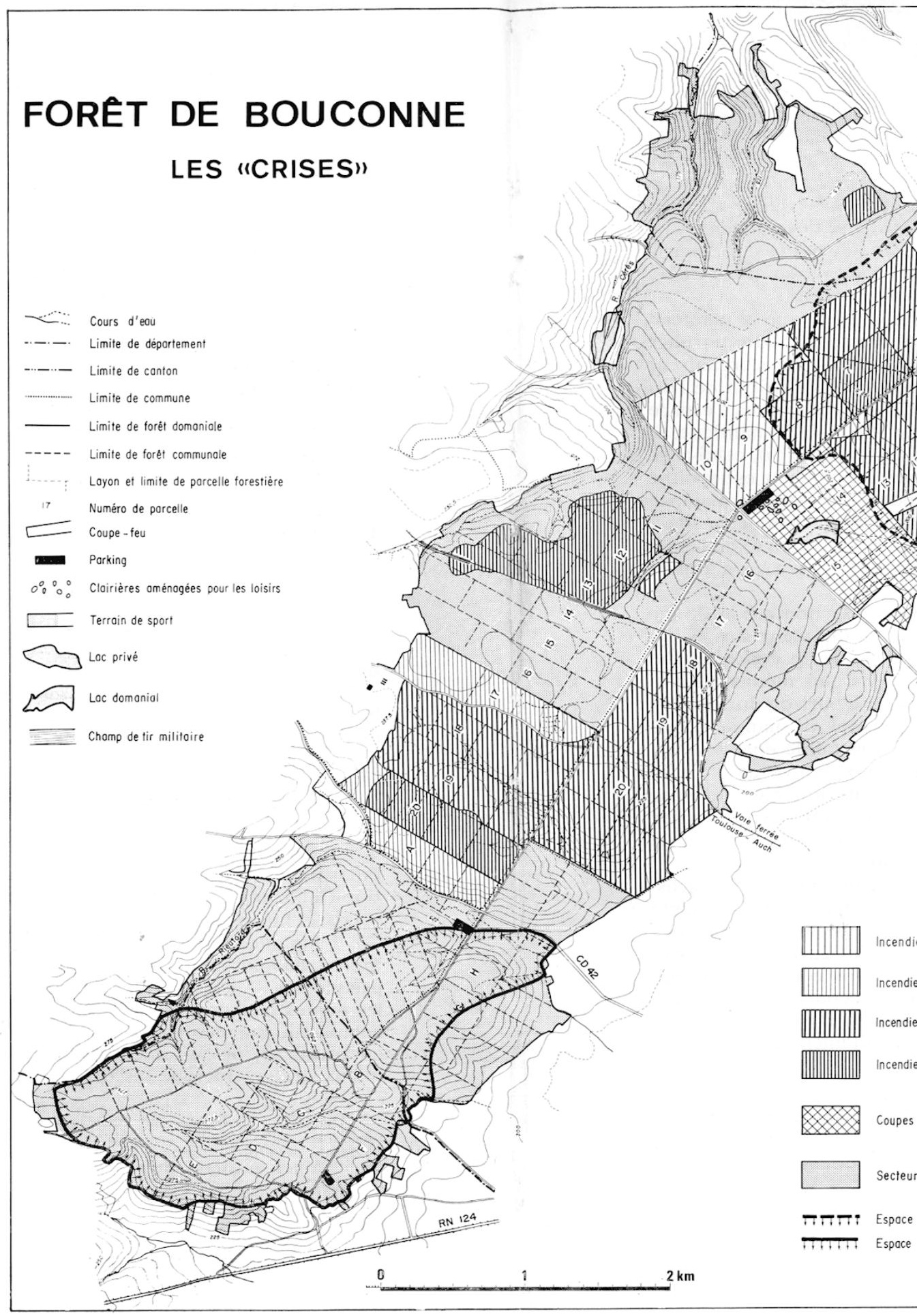


Sols bruns lessivés et surtout sols bruns modaux ne couvrent qu'une superficie très restreinte : versants des vallons et talus des terrains, à pente relativement forte. Ils se présentent notamment en petites plages au sud de la forêt, dans le bois de Lévignac au nord et au NW du carrefour principal de la D 24. Ils se développent d'autant mieux que, dans ces secteurs, les remaniements superficiels sont fréquents et les dépôts plus récents, donc moins différenciés (les sols bruns modaux représentant un cas extrême).

Même sur pente déjà marquée, mais à condition que le manteau limoncux ne soit pas trop mince, les sols lessivés hydromorphes modaux ne sont pas rares. Cependant ces profils ne s'organisent régulièrement que là où la pente reste modérée. Il s'agit là de l'orthotype pédologique de la forêt, auquel s'associent dans l'espace toutes les variantes possibles. Du point de vue hydrodynamique, le type modal ne se différencie guère du type glossique (6), celui-ci se développe très généralement là où la tranche limoneuse est épaisse et parfois même en secteur à très faible pente. La variante planosolique, en revanche, témoigne d'une ambiance hydromorphe déjà plus grande - et moins favorable à l'arbre - : elle couvre de larges secteurs des éléments de plateaux, notamment sur $\mathrm{T} 2$, au centre de la forêt.

Les pseudogleys et les sols hydromorphes à accumulation de fer (grep) sont souvent associés dans l'espace, notamment sur T3, au Nord de la D24. Du point de vue forestier, si les pseudogleys ne sont pas des plus favorables, les formations les plus médiocres correspondent sans aucun doute aux sols à greps. L'horizon dur concrétionné par le fer, apparenté à un cuirassement de nappe, ne se forme qu'au sommet du cailloutis et sous une faible épaisseur de limon, par imprégnation saisonnière liée aux battements de la nappe phréatique perchée. Ces sols cumulent pratiquement tous les inconvénients : barrière mécanique pour les racines; plancher imperméable qui aggrave l'engorgement par l'eau; accentuation de la sècheresse estivale par blocage de la capillarité ascendante.

Ainsi esquissée, cette typologie ne donne qu'une idée encore grossière de l'aptitude forestière des sols. D'une part, en effet, il n'existe pas de cartographie opérationnelle (à 1/10 000, par exemple) des sols de la forêt; en outre, l'arrangement spatial du manteau pédologique, aux grandes échelles, est extrêmement complexe en raison notamment de la fréquente organisation en courtes toposéquences et de la variabilité des structures alluviales. Il en résulte, d'autre part, que la taxonomie utilisée - parfaitement accordée, au

6. Toutefois, les sols glossiques étant nécessairement plus "profonds », ils nc présentent pas l'inconvénient assez fréquent des sols lessivés hydromorphes modaux établis sur limons moins épais : la légère induration du sommet de la nappe caillouteuse, provoquée par les petites concrétions et revêtements ferro-manganiques, gếne en effet la pénétration des racines et les échanges hydriques. 
demeurant, à des échelles de l'ordre de 1/50 000 et au-delà - est ici impuissante à rendre compte du comportement réel des peuplements forestiers, face à une variable aussi mouvante que le bilan hydrique des profils. Sans doute la corrélation biomasse - type de sol est-elle acceptable pour les catégories extrêmes : ainsi pour les sols bruns modaux et bruns lessivés, à forte croissance; pour les pseudogleys et les sols à grep, à croissance lente. Mais le groupement majoritaire à sols lessivés hydromorphes modaux, glossiques et planosoliques - ces derniers toutefois déjà plus proches des pseudogleys - rassemble à l'évidence des secteurs où la croissance de la biomasse varie sensiblement (fig. 1). Il est clair que, dans ce cas, des paramètres nous échappent. Indépendamment d'autres facteurs - traitements antérieurs, effets d'inertie ou de proximité, etc. - on peut penser que l'épaisseur des limons et le rythme piézométrique des profils jouent un certain rôle. Une étude à grande échelle de ces variables permettrait sans aucun doute d'affiner le système de corrélations.

Il reste qu'en définitive et pour l'essentiel, la forêt de Bouconne se compose de deux grands milieux morpho-pédologiques.

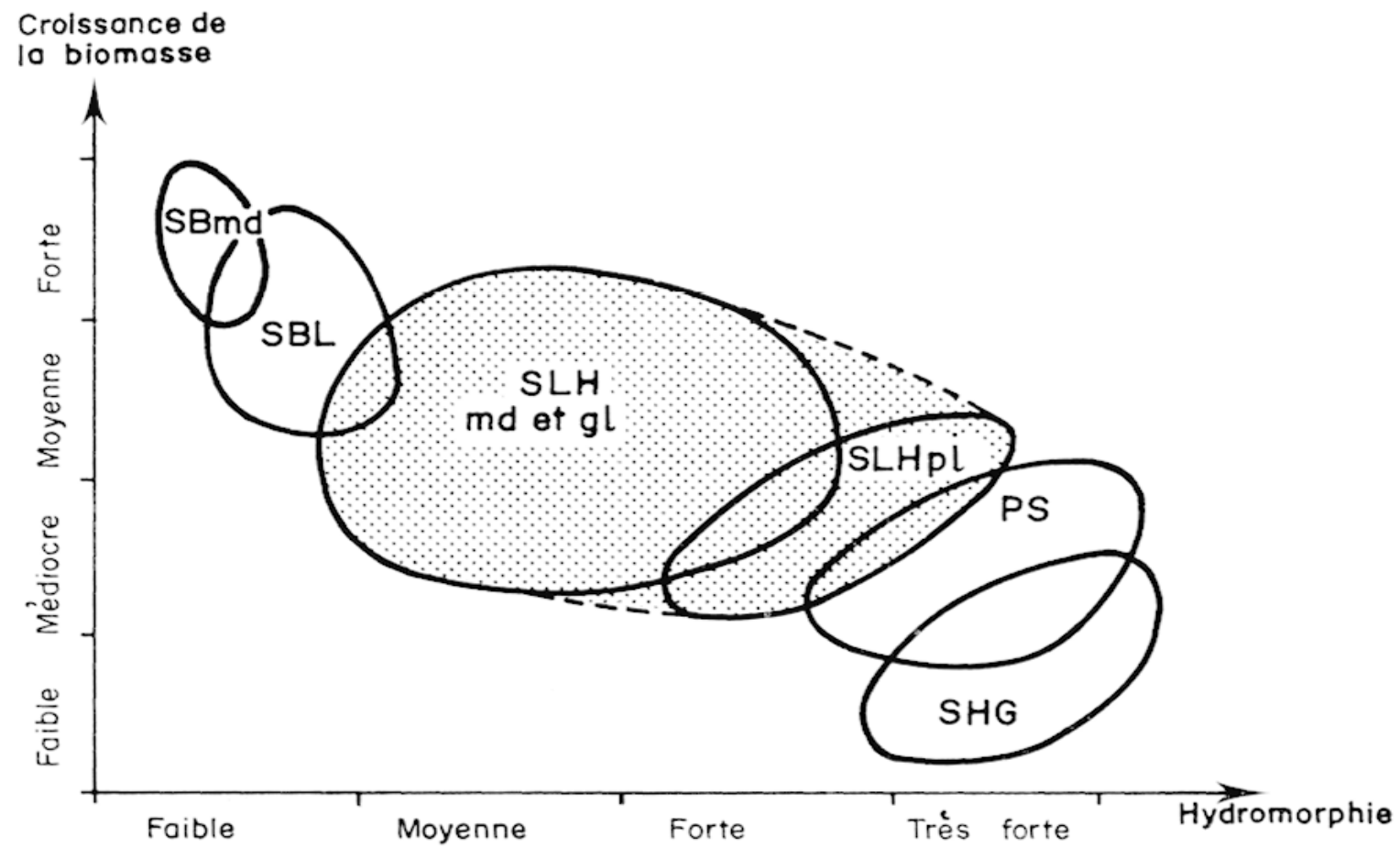

FIG. 1

Relations entre types de sols, hydromorphie et croissance de la biomasse.

SB : Sol brun modal. - SBL : Sol brun lessivé. - SLH : Sol lessivé hydromorphe. - md : modal. - gl : glossique. - pl : planosolique. — PS : Pseudogley. - SHG : Sol hydromorphe à accumulation de fer (grep). 
D'une part, les surfaces planes des terrasses, à drainage médiocre ou franchement déficient : l'existence d'une faible pente, la proximité des vallons ou talus, l'épaisseur du limon et la présence d'horizons à grep, y déterminent un ensemble de facettes souvent étroitement imbriquées.

D'autre part, les versants, vallons, talus de terrasses, Iongs glacis raccordant les paliers entre eux : les sols y sont en général bien mieux ressuyés. Pseudogleys et formations à grep en sont absents.

Les terrasses sont d'autant plus disséquées et démantelées qu'elles sont plus anciennes, aussi l'cspace forestier sc trouve naturcllcment divisé en deux grands secteurs. Le premier milieu, médiocre, s'étend largement au nord et au centre; le second, de meilleure venue, occupe la partie sud, beaucoup plus vallonnée. Du contraste entre ces deux profonds milieux naît l'opposition entre les parcelles méridionales, d'allure véritablement forestière, et celles du Nord, couvertes de mauvais taillis et de landes. Cette dichotomie du tissu forestier ne tient cependant pas uniquement au substrat : les modalités de la gestion forestière, en particulier, ne sont pas restées sans effet.

\section{Gestion, exploitation et crises : le passé récent}

Comme presque toute la forêt française, Bouconne doit aux hommes son statut, ses limites et nombre de ses caractères actuels; (7). C'est seulement à partir des années 1830 cependant, que débute véritablement l'aménagement forestier (8). De 1835 à 1850, Laby de Vaumont, sous-inspecteur des Forêts de la résidence de Toulouse, fait établir un réseau de fossés d'assainissement. Un siècle plus tard, en 1950, on élargit et approfondit certains de ces canaux, et on en creuse de nouveaux, essentiellement sur $\mathrm{T} 3$ (série $\mathrm{I}$, parcelles 1 à 8 ; série II, parcelles 1 à 12), cette partie étant parfois localement inondée.

Parallèlement à cela, la forêt est divisée en séries et parcelles (1947) pour permettre une exploitation et un aménagement plus rationnels. L'espace est partagé en trois séries. L'une d'elles, au sud (407 ha) est divisée en dix coupes désignées par des lettres allant de $A$ à $\mathrm{J}$, (le bois de Lisle, au sud-est, qui appartient à la commune de Lisle-Jourdain, est lui aussi divisé et soumis à la gestion de l'O.N.F.). Les deux autres couvrent le reste de la forêt; la série I, dite de Lasserre, (812 ha) à l'ouest de l'allée forestière centrale, divi-

7. S. HENRY : La forêt de Bouconne. Etude de géographie historique. Toulouse, 1943, Privat.

8. J. Mallet et J. Liwerant, Bouconne, forêt dégradée du pays toulousain, Revue forestière française, $\mathrm{n}^{\circ} 11,1956$. 
sée en 20 coupes numérotées de 1 à 20 ; et la série II, dite de Pibrac (728 ha) à l'est, divisée elle aussi en 20 coupes. Dans ces deux séries, qui en 1947 étaient réduites à un maigre taillis piqueté de quelques Chênes de belle taille, les forestiers préconisèrent un traitement en taillis sous futaie. La révolution des coupes était de 30 ans (sauf pour une partie des séries mises en réserve). La série sud, au contraire, fut traitée en futaie jardinée. Entre-temps, depuis 1880 , on y avait introduit le Pin sylvestre et le Pin maritime en mélange avec le Chêne. On procédait alors par trouées autour de porte-graines ou par éclaircie en abattant les arbres les moins beaux. Cet aménagement favorisait ainsi la venue d'une belle forêt là où les arbres poussaient déjà bien : choix parfaitement légitime... A partir de 1954 cependant, voyant que les taillis des séries de Lasserre et Pibrac croissaient toujours aussi lentement, on décida de les enrésiner à leur tour.

Mais il fallait compter aussi avec parasites et incendies. En 1903, les Chênes sont attaqués par l'Ö̈dium. La chênaie défeuillée n'ombrageant plus le sol, un sous-bois acidiphile touffu put ainsi se développer, gênant la croissance des plantules d'arbres. Peu de temps après, c'est au tour des Pins sylvestres d'être parasités; on les remplaça peu à peu par des Pins maritimes plantés sur T3 qui sont attaqués par un champignon, le Cronartium, puis par un insecte, le Pissode, et tous dépérissent. A ces crises s'ajoutent des prélèvements incontrôlés: la forêt est soumise pendant les deux dernières guerres mondiales à de fortes coupes de bois $\left(8000 \mathrm{~m}^{3}\right.$ en 1914-1918 et $5000 \mathrm{~m}^{3}$ en 1939-1945) beaucoup trop rapprochées pour des arbres à croissance limitée. De plus, à ces mêmes périodes, des incendies particulièrement fréquents ravagent les surfaces planes où les arbres poussent lentement et où le sous-bois reste très dense. Entre 1918 et 1924, ces incendies successifs, consécutifs aux coupes de la Première guerre mondiale, détruisent 770 ha au nord de la forêt dans un premier temps, puis 450 ha en 1923-1924. Entre 1918 et 1924, les parcelles 1 à 8 de la série de Pibrac ont brûlé deux fois. Une nouvelle série d'incendies se déclenche en 1945 sur T2 alors que les parcelles 5 à 7 de la série I et 13 à 15 de la série II sont surexploitées. Notons ici que toutes ces coupes intempestives ne touchent pas la futaie du sud de la forêt. Entre 1949 et 1953, les incendies sont encore très rapprochés et l'un d'eux, au mois de mars 1949 détruit 360 ha de forêt sur T3. De 1960 à 1964 les passages du feu sont plus rares : ils sont exceptionnels depuis (Carte, p. 228-229).

Cette longue série de désastres, pèse d'un poids très lourd dans la physionomie actuelle de la forêt. Toutefois, celle-ci n'a pas été touchée également dans toutes ses parties. Maladies cryptogamiques et pullulations d'insectes ont affecté en priorité le nord et le centre de la forêt, là où la médiocrité du drainage et du substrat détermine des peuplements peu vigoureux. De plus, l'ambiance humide de ces milieux favorise à coup sûr le développement des 
parasites. Mais ce sont surtout les passages répétés du feu qui, épargnant la partie sud, ont ravagé le reste de la forêt. C'est que les incendies se propagent plus aisément en terrain plat, balayé par le vent, que dans les secteurs vallonnés. En outre, ils affectent en priorité les parcelles couvertes de mauvais taillis et de peuplements généralement encore jeunes et mal venus, qui forment la majeure partie du tissu forestier au nord et au centre. Ainsi, par exemple, sur T3 : en 1918, les individus de parcelles incendiées sont âgées de 6 ans; en 1924, de 6 à 10 ans; en 1949 (série I, parcelles 6 et 7; série II, parcelles 13 et 14), les Chênes atteints n'ont pas dépassé 4 ans et ceux qui brûlent en 1952 et 1960 n'ont pas 10 ans. On pourrait multiplier les exemples : dans l'ensemble, il s'agit de jeunes taillis où les Chênes se mêlent aux plantes de la lande (notamment Genêts et Bruyères) en masses confuses et touffues. Tous ces incendies anéantissent bien entendu les plantations de jeunes Pins. Par la suite, le taillis de Chênes se régénère par rejets de souche, mais l'éclaircissage brutal occasionné par le feu - tout comme celui qui résulte de coupes excessives - entraîne le développement momentané de larges plages de lande acidiphile... et le cycle peut recommencer.

On peut retenir de tout cela un point capital : en dépit des aménagements, l'aspect et la dynamique des peuplements forestiers de Bouconne restent étroitement tributaires des grandes variables naturelles du substrat : pente, régime hydrique, sols. Plus encore, la succession des opérations d'aménagements et des crises n'a fait que renforcer, pour l'essentiel, l'opposition naturelle entre bons et mauvais secteurs. De ce fait majeur, la cartographie intégrée de la forêt de Bouconnc devait tenir compte en priorité.

\section{Les géofaciès (carte hors-texte)}

Pour définir et classer les milieux forestiers (désignés sous le nom de géofaciès, fractions constitutives d'un même géosystème forestier) on s'est appuyé à la fois sur la physionomie actuelle des peuplements et sur leur dynamique, exprimée par la vitesse d'accroissement de la biomasse (cf. tableau 1). Ce dernier paramètre était relativement facile à apprécier dans une forêt comme Bouconne, dont toutes les opérations d'aménagement, les plantations ou les coupes ont été soigneusement enregistrées depuis longtemps. Une fois connu l'âge des arbres, on a donc pu estimer rapidement leur vitesse de croissance. De là découle le classement des géofaciès en quatre catégories majeures : géofaciès arbustifs et arborescents, à croissance lente ou très lente; géofaciès subforestiers, à croissance médiocre; géofaciès forestiers, à croissance moyenne ou rapide. Ils seront sommairement présentés un peu plus loin. (Cf. carte h.t.). 


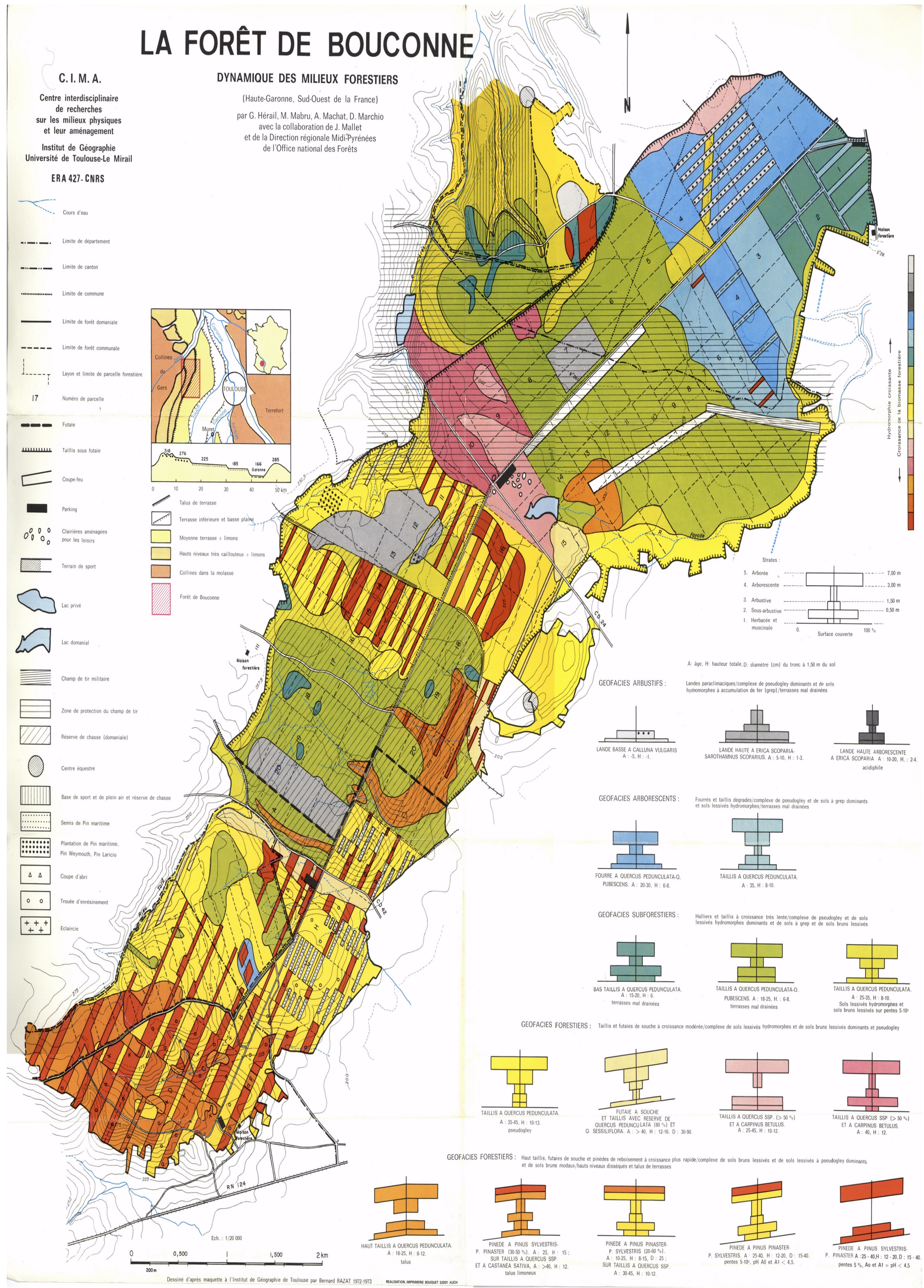


Une représentation suffisamment claire et lisible de ces géofaciès imposait le choix d'un principe directeur qui fût à la fois simple et de valeur discriminante générale. On a retenu le régime hydrique des sols, ou, ce qui revient au même, le degré d'hydromorphie du substrat, facteur qui intègre très largement les variables topographiques et pédologiques. Ainsi, entre les pôles extrêmes: futaies bien venues des secteurs bien drainés et taillis ou landes des zones très hydromorphes, se distribue toute une séquence de géofaciès intermédiaires facilement repérables sur la carte. Ce repérage est donné par une gamme de couleurs qui, des tons froids (gris, bleu) aux tons chauds (rose, rouge) progresse parallèlement au drainage et à la croissance des peuplements. Pour chaque géofaciès, la légende comporte une pyramide structurale : nombre et importance relative des strates, hauteur et âge des arbres, etc. Le tracé de la base de ces pyramides schématise par ailleurs l'assiette topographique (plateau, versant ou contact) des géofaciès. Notons enfin que, dans ces derniers, la vitesse de croissance des strates n'est pas toujours homogène, comme l'indiquent les pyramides bicolores.

\section{Les géofaciès arbustifs.}

La végétation de ces milieux est celle d'une lande où la reconquête se fait d'elle-même par rejet ou repousse des arbres détruits exception faite des plantations ou des semis. Ces géofaciès représentent une phase postérieure à la dégradation forestière et antérieure à la reconquête. La première formation qui se met en place est une lande basse (1 mètre de haut) composée d'abord de Graminées, de Ronces et de Genêts à balai. Très vite (3 à 4 ans), la Callune et la Bruyère à balai envahissent ce milieu. Après 5 ans, le faciès change et on passe à une lande haute piquetée de quelques pousses de Chêne pédonculé dominées par la Bruyère à balai et le Genêt à balai. Il faut attendre une dizaine d'années pour que l'importance de la strate herbacée et sous-arbustive diminue relativement. Ces géofaciès caractérisent des milieux mal drainés à sols très hydromorphes et cuirassements ferrugineux (grep) fréquents.

\section{Les géofaciès arborescents.}

Deux types peuvent être distingués : le fourré à Chêne pédonculé et à Chêne pubescent et le taillis à Chêne pédonculé légèrement plus haut et à stratification différente. Mais dans les deux cas la strate arborée est ouverte et basse (environ 8 mètres) et la croissance très lente. Ces géofaciès se développent sur des topographies planes ou même légèrement déprimćes où l'écoulement est malaisé; les sols sont du type lessivé hydromorphe ou pseudogley. 


\section{Les géofaciès subforestiers.}

Trois types ont été distingués; parmi tous ces taillis poussant très lentement sur des sols le plus souvent fortement hydromorphes, celui à Chêne pédonculé et Chêne pubescent, est très représentatif. Les pousses âgées de 18 à 25 ans, ne dépassent pas 6 à 8 mètres de haut, les souches portent 3 à 6 brins d'un diamètre inférieur à 10 centimètres et sont espacées de 2 à $4 \mathrm{~m}$. Ce taillis peut pousser sur des limons relativement épais $(80 \mathrm{~cm}$ à $100 \mathrm{~cm})$. Lorsque le drainage se fait plus difficile, on passe à un taillis bas de Chêne pédonculé et sur les légères pentes, au contraire, le taillis est plus élevé et les sols plus sains. Ces géofaciès qui couvrent de grandes surfaces sur les terrasses $\mathrm{T} 3$ et $\mathrm{T} 2$ portent la marque des grands incendies qui ont eu lieu entre 1945 et 1953. La reconquête a été difficile sur un substratum peu favorable, mais actuellement la strate arborée, basse, est presque fermée et entrave le développement d'un sous-bois à espèces héliophiles acides.

\section{Les géofaciès forestiers.}

Ils sont représentés par des taillis, des taillis sous futaie ou des futaies de souche poussant sur des sols lessivés hydromorphes, ou sols bruns lessivés peu hydromorphes et localement au Sud, sur des sols bruns modaux. Dans l'ensemble, la croissance de la biomasse est nettement meilleure. Physionomiquement, ces géofaciès se distinguent clairement des géofaciès subforestiers : ici, la strate arborée domine toujours dans les pyramides. Les géofaciès forestiers peuvent être rassemblés en deux groupes: ceux dont les arbres croissent de façon modérée et ceux à croissance plus rapide.

Au premier groupe appartiennent les taillis à Chêne pédonculé, la futaie et taillis à Chêne pédonculé et Chêne sessile et les taillis à Charme et Chêne. La présence d'un taillis sous futaie à Chêne pédonculé et Chêne sessile est remarquable. Le taillis de Chêne, composé d'arbres des deux espèces, est âgé de 28 à 46 ans et atteint 10 à 12 mètres; les arbres de la futaie ont de 80 à 150 ans. Ces peuplements ont été épargnés par les incendies récents et par les maladies graves; de plus, la pente atténue les effets néfastes de l'hydromorphie et en bout de versant les sols limono-caillouteux assurent l'évacuation des eaux. La présence du Charme permet d'individualiser deux géofaciès forestiers originaux.

Dans le deuxième groupe, il faut ranger le haut taillis à Chêne pédonculé, les géofaciès associant résineux et feuillus et enfin les pinèdes pures. Le taillis à Chêne pédonculé, comme d'ailleurs les taillis à Chêne et Châtaignier coiffés par une pinède à Pin sylvestres et Pin maritime, se développent sur des topographies disséquées où les sols bruns lessivés ne sont pas rares. Les arbres poussent plus vite et dépassent de 2 à 3 mètres les taillis de même âge 
ou parfois plus vieux, établis à proximité sur des substrats plus médiocres. Le long et minutieux travail des forestiers s'exprime ici mieux que partout ailleurs et constitue un des facteurs de cette dynamique. Il faut toutefois remarquer que lorsque les Pins couvrent plus de $40 \%$ de la strate arborée, les Chênes semblent éprouver quelque difficulté à se maintenir. Leur position en sous-bois, l'ombre et l'atmosphère qui y règnent, l'acidification plus poussée du sol ( $\mathrm{pH}<4,5$ dans les horizons Ao et $\mathrm{Al}$ ) ne leur sont pas favorables. Aussi peut-on penser que le jardinage favorise la création des pinèdes pures.

Le géofaciès à pinède pure est celui où la croissance est la plus rapide. Les arbres, Pin sylvestres ou Pins maritimes, âgés de 25 à 40 ans, hauts de 12 à 20 mètres, développent un tronc dont le diamètre est compris entre 15 et $40 \mathrm{~cm}$. Cette pinède est l'aboutissement de la politique forestière mise en œuvre dans la partie sud de la forêt. L'élimination des Chênes, la modification et l'appauvrissement du sous-bois, l'acidification du sol sont les conséquences normales de cette évolution au demeurant contrôlée par les forestiers. Ceux-ci tendent aujourd'hui à maintenir les feuillus dans les formations résineuses. 
RÉsumÉ. - La forêt de Bouconne, située sur les hautes terrasses de la Garonne à l'ouest de Toulouse, est un taillis de Chênes piqueté de Pins. La distribution des divers géofaciès forestiers et la croissance de la biomasse forestière sont fonction de la qualité des sols (surtout de leur drainage) d'une part, de la gestion et des crises (incendies, parasites, coupes parfois très rapprochées) d'autre part. Mais en dépit des aménagements l'aspect et la dynamique des peuplements forestiers restent étroitement tributaires des grandes variables naturelles et surtout du régime hydrique des sols. Ces contrastes ont été renforcés par la succession des opérations d'aménagement et c'est le parallélisme entre le bon drainage du sol et la rapidité de croissance des peuplements qui est la clef de la légende de la carte.

Summary. - The Bouconne forest near Toulouse. An essay of an Intrgrated MAPPING OF A WOODLAND AREA. Grown west of Toulouse on the highest alluvial terraces built up by the Garonne river, the Bouconne woodland area is commonly known as a forest when it is an oak taillis dottered with pine trees. The spatial distribution of the various woodland geofacies and the growth of the aggregate vegetal biomass as well depend on one hand on the soils' nature (and mainly on their drainage) and on the other hand on the economic management of the woodland and the various crisis that it met (wood fires, invasions of parasites or too frequent cutting down of timber on the same plot). Drainage and timber growth quickness are the two major keywords for the map.

RESUnies. - La "forêt de Bouconne " : un mapa forestal. La " Forêt de Bouconne" situada sobre las altas terrazas del río Garona, al oeste de Toulouse, es un monte alto de robles salpicado de pinos. La distribución de los diversos geofacies forestales y el crecimiento de la biomasa forcstal están relacionados por una parte con la calidad de los suelos (esencialmente el drenaje) y por otra parte con la gestion y las crisis (incendios, parásitos, cortes a veces muy frecuentesi. Pero a pesar de las ordenaciones, el aspecto y la dinámica de las poblaciones forestales quedan estrechamente ligadas a las grandes variables naturales y sobre todo el regímen hídrico de los suelos. Estos contrastes han sido acentuados por una serie de operaciones de ordenación y el paralelo que existe entre un buen drenaje del suelo y la rapidez del crecimiento de los árboles ha servido de clave para la legenda del mapa. 